\title{
Tocotrienol-rich vitamin E from palm oil (Tocovid) and its effects in diabetes and diabetic retinopathy: a pilot phase II clinical trial
}

\author{
Yilynn Chiew ${ }^{1,2}$, Suzanne May Quinn $\operatorname{Tan}^{1,2}$, Badariah Ahmad ${ }^{1,2}$, Sim \\ Ee Khor ${ }^{3}$, Khalid Abdul Kadir ${ }^{1,2}$ \\ 'School of Medicine and Health Sciences, Monash University Malaysia, Selangor, \\ Malaysia; ${ }^{2}$ Tropical Medicine and Biology Platform, School of Science, Monash \\ University Malaysia, Jalan Lagoon Selatan, Bandar Sunway, Selangor, Malaysia; \\ ${ }^{3}$ Thomson Hospital Kota Damansara, Petaling Jaya, Selangor; Malaysia
}

\begin{abstract}
Aim: To identify the effects of tocotrienol-rich vitamin E from palm oil (Tocovid) on diabetic retinopathy $(D R)$ in patients with type 2 diabetes.

Materials and methods: The intervention group $(n=21)$ received $200 \mathrm{mg}$ Tocovid twice daily while the control group $(n=22)$ received placebo twice daily for 8 weeks. Changes in retinal photography by conventional grading and novel quantification of retinal hemorrhage were assessed. Changes in serum biomarkers advanced glycation end products (AGE) general, sRAGE (soluble receptor of $A G E$ ), NE-CML (specific type of $A G E)$, and cystatin $($ were evaluated.

Results: A novel technique to quantify retinal hemorrhage had a strong positive correlation with conventional grading of $D R$ in both eyes at baseline and at the end of the study. Eight-week supplementation of Tocovid resulted in significant reduction in retinal hemorrhage in the right eye. Liver enzymes and ALT significantly reduced. No significant changes in grade of $\mathrm{D} R$, serum biomarkers, $\mathrm{HbA1C}$, blood pressure, renal profile, and lipid profile were observed.

Conclusions: Tocovid is a potential adjunct to current treatment of DR and fatty liver disease. A novel method of quantifying retinal hemorrhage is a potential technique for assessing disease severity of $D R$, particularly the early changes.
\end{abstract}

Keywords: advanced glycation end products, carboxymethyl-lysine, diabetic retinopathy, palm oil, tocotrienol, vitamin $E$

Correspondence: Dr. Yilynn Chiew, School of Medicine and Health Sciences, Monash University Malaysia, Selangor 46150, Malaysia.

E-mail:drchiewyilynn@gmail.com 


\section{Introduction}

Diabetes mellitus (DM) is an alarming public health concern that is approaching epidemic proportions on a global scale. Diabetic retinopathy (DR), one of the main microvascular complications of diabetes, affects $34.6 \%$ of the diabetic cohort. ${ }^{1}$ It is a leading cause of new onset blindness among individuals aged 20 to 64 years old and accounts for $2.6 \%$ of global blindness. ${ }^{2,3}$ Effective treatment strategies to prevent onset and progression of DR are needed.

Vitamin $\mathrm{E}$ has been touted as a potent antioxidant capable of attenuating oxidative and inflammatory stressors involved in the pathogenesis of diabetes and its related complications. It exists in two major forms, tocopherol and tocotrienol. Its therapeutic role in diabetes, however, remains largely controversial. ${ }^{4-6}$ The bulk of the studies done on vitamin $\mathrm{E}$, however, is on the more common tocopherol. Tocotrienol on the other hand, only contributes to less than $3 \%$ of the entire literature on vitamin E. Interestingly, growing evidence has demonstrated that tocotrienols possess antioxidant properties that are 40-60 times greater than tocopherols and is more capable of ameliorating diabetic-related complications. ${ }^{7}$ A study by Nazaimoon et al. demonstrated that tocotrienol was able to reduce lipid peroxidation within 2 months in patients with type 2 DM (T2DM). ${ }^{8}$ Other animal studies further endorse the antioxidative properties of tocotrienol in diabetic rat models. ${ }^{9-12}$ Among the many sources of tocotrienols, vitamin E extracted from palm oil (Elaeis guineensis) contains the most concentrated form of tocotrienols. ${ }^{13,14}$

In addition, animal studies have shown that tocotrienols possess antiangiogenetic abilities and confers significant protection in the retina. ${ }^{15,16}$ However, literature on the effects of tocotrienol in attenuating DR is limited. Nevertheless, tocotrienol has also been shown to reduce advanced glycation end products (AGE), a key component in the pathogenesis of DR. ${ }^{9,17}$ AGE constitute a group of inflammatory biomarkers formed from reactions between reducing sugars and free amino acids, nucleic acids, and lipids. ${ }^{18}$ AGE react with its receptor, RAGE, to produce oxidative stress and activate proinflammatory cytokines including RAGE itself, thus forming a vicious cycle. This ultimately results in DR and other diabetic-related complications. ${ }^{18,19}$ On the same note, the landmark Diabetes Control and Complications Trial/Epidemiology of Diabetes Interventions and Complications Trial (DCCT/EDIC) and United Kingdom Prospective Diabetic Study (UKPDS) also demonstrate that it is not $\mathrm{HbA1C}$, which lasts only 3 months, but AGE, which last a lifetime, that are better predictors of DR. ${ }^{20-25}$ As the current treatment for DR, which is tight glycemic control, does not address the AGE pathway, tocotrienol is proposed as an adjunct to the treatment regime of DR.

Among the $A G E, N$-epsilon-carboxymethyl lysine $(\mathrm{N} \varepsilon-\mathrm{CML})$ has been shown to be the most promising biomarker for predicting the presence and 
progressive severity of DR. ${ }^{20,26-28}$ Another potential biomarker for DR is soluble RAGE (sRAGE). ${ }^{29,30}$ sRAGE isoforms sequester AGE and therefore ameliorate against AGE-RAGE mediated complications of diabetes. ${ }^{31}$ On the other hand, cystatin $C$ is a novel biomarker which has been shown to be superior to serum creatinine in detecting the early stages of diabetic nephropathy, another microvascular complication of diabetes. ${ }^{32}$

Therefore, the primary aim of this pilot study is to investigate the effect of tocotrienol-rich palm oil (Tocovid) compared with placebo on inflammatory biomarkers $\mathrm{AGE}$, sRAGE, $\mathrm{N} \varepsilon-\mathrm{CML}$, and cystatin $\mathrm{C}$ and on the disease severity of DR as assessed by retinal photography. The secondary aim of this pilot study is to investigate the effect of Tocovid on other glycemic and metabolic parameters in T2DM.

\section{Materials and methods}

\section{Study design}

This study was designed as a prospective, randomized, double-blinded, placebo-controlled clinical trial to compare the effect of Tocovid versus placebo in adult T2DM patients with DR. This study consisted of 8 weeks of screening and 8 weeks supplementation with either Tocovid or placebo. The overall study design is shown in Figure 1. Ethical approval was obtained from the Monash University Human Research Ethics Committee (Project number: 12091) and the study complied with the principles of the Declaration of Helsinki. Informed written consent was obtained from all participants prior to commencement of screening. The sample size was set at 52 participants based on previous literature. ${ }^{33}$ This was based on power calculations demonstrating a difference of $30 \% \pm 5 \%$ SD in primary outcome between the intervention and control group. However, due to financial constraints, only 43 participants were recruited.

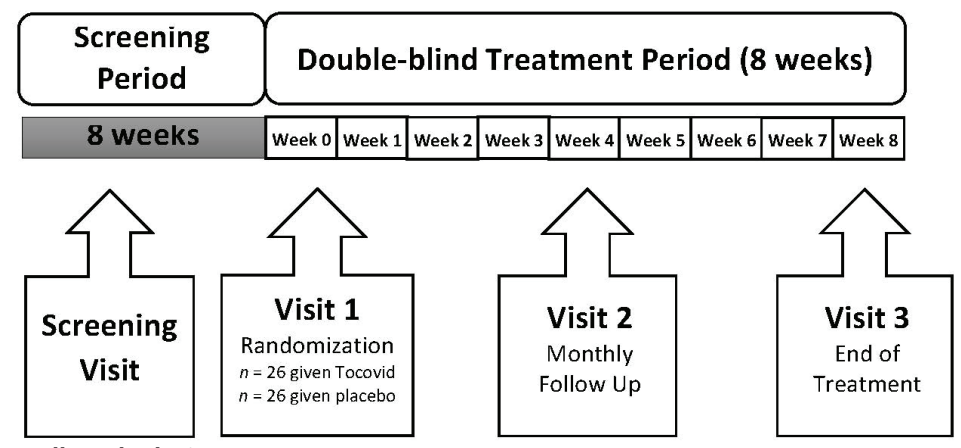

Fig. 1. Overall study design. 


\section{Participants and study enrolment}

Participants were recruited from the existing pool of patients at the Monash University Clinical Research Center (CRC) in Johor Bahru and in Bandar Sunway, Malaysia. Patients with T2DM were invited to attend the screening visit. Patients were reminded to observe an $8 \mathrm{~h}$ fasting period, as per routine diabetes follow-ups, to miss their morning dose of diabetic medication, not to wear contact lenses, and bring their prescribed visual aids.

During the screening visit, complete history-taking and physical examination was obtained. Further screening tests including fasting blood glucose, $\mathrm{HbA} 1 \mathrm{c}$, and digital retinal photography were obtained. Safety tests including electrocardiogram, renal profile, liver function test, lipid profile, and urine dipstick were conducted to confirm the participants' fitness to participate in the study.

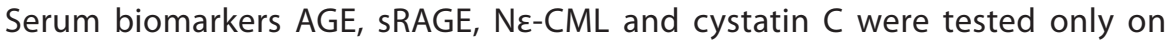
patients who met the inclusion and exclusion criteria.

Participants with the following criteria were included:

1. Participants aged $18-80$ years old at the time informed consent was provided.

2. Participants who had T2DM with stable glucose control (not more than $10 \%$ change in $\mathrm{HbA} 1 \mathrm{c}$ levels over the last 2 months) and $\mathrm{HbA} 1 \mathrm{c}$ range within $6.5-14 \%$.

3. If the participant had hypertension, they must have had stable blood pressure control (not more than 10\% change in blood pressure over the last 2 months) and blood pressure range of less than $160 / 100 \mathrm{mmHg}$.

4. Participants who had stable DR in at least one eye as assessed by retinal photography with no laser, intraocular injections, or other invasive treatment in the last 3 months.

Participants with the following criteria were excluded from the study:

1. Participants who had severe media opacity (i.e., corneal opacities, dense cataracts, and vitreous hemorrhage).

2. Participants who had acute or severe eye diseases (i.e., retinal detachment, periorbital cellulitis, and angle-closure glaucoma).

3. Participants who had acute or severe chronic diseases (i.e., acute coronary syndrome, active tuberculosis, active cancer, end-stage kidney failure, liver or inflammatory diseases).

4. Participants who were pregnant or hoped to become pregnant during the trial.

5. Participants who took water-soluble antioxidants (i.e., vitamins B or $C$, polyphenols, glutathione) during the past 2 weeks or fat-soluble 
antioxidants (i.e., vitamins A, D, E and K) during the past 4 weeks.

6. Participants who were heavy smokers ( $\geq 20$ sticks/day) or had stopped smoking for less than 1 month.

\section{Randomization, study groups, and supplement formulation}

Participants who met the inclusion and exclusion criteria were randomized into double-blind treatment period for 8 weeks. Randomization was done by an independent operator by using computer-generated permuted blocks of size 4 in a 1:1 ratio for intervention or control, stratified for patient's age, gender, and duration of DM. Allocation concealment was done by sequentially numbered opaque sealed envelopes.

Both Tocovid and placebo capsules were of similar shape, size, taste, and excipients. The study drugs were labelled as Drug A and Drug B and their identity was kept confidential by ExcelVite (Hovid Berhad, Ipoh, Malaysia). Allocation was concealed until the end of the study. The intervention group received $200 \mathrm{mg}$ twice a day tocotrienol-rich vitamin E (Tocovid Suprabio ${ }^{\mathrm{TM}}$ ), while the control group received placebo twice a day for 8 weeks. EVNol SupraBio, the active ingredient of Tocovid Suprabio ${ }^{\mathrm{TM}}$ was manufactured by ExcelVite, Malaysia.

\section{Follow-up visits}

Follow-up visits every 4 weeks were conducted to monitor for adverse drug events and participant's compliance to treatment via capsule count. Anthropometric measurements, blood pressure, fasting blood glucose, and urine dipstick were routinely carried out during follow-up visits. In the final visit, $\mathrm{HbA} 1 \mathrm{c}$, retinal photographs and biomarkers AGE, sRAGE, NE-CML and cystatin $C$ were obtained. Baseline safety tests including ECG, renal profile, liver function test, and lipid profile were repeated to ensure the study drugs had no adverse effect to the participant.

\section{Evaluation of outcomes}

The primary outcomes were biomarkers AGE, sRAGE, NE-CML, and cystatin C and the disease severity of DR as assessed by retinal photography. The secondary outcomes were $\mathrm{HbA} 1 \mathrm{c}$, blood pressure, and other safety tests including renal, lipid, and liver profile.

\section{Retinal photographs}

Retinal photographs were taken using a fully automated, non-mydriatic digital retinal imager (Digital Retinography System (DRS), CenterVue, Padova, Italy). Seven different $45^{\circ}$ fields were taken for both eyes using this retinal camera at baseline and after 8 weeks. Thereafter, high quality multi-image mosaics (retinal maps) were constructed from the 7-field retinal photographs using an 
advanced image registration and montage software i2k Retina ${ }^{\circledR}$ software (Dual Align LLC, Clifton Park, NY, USA) to obtain a more complete and accurate view of the patient's retina. ${ }^{34}$ The retinal mosaics or maps were then graded for DR based on the Early Treatment Diabetic Retinopathy Study (ETDRS) grading ${ }^{35}$ by an ophthalmologist who was double-blinded. Participants were allocated one of five retinopathy grades: no DR (NDR), mild non-proliferative DR (NPDR), moderate NPDR, severe NPDR, and proliferative DR (PDR).

Additionally, the retinal maps were quantified manually by the investigator and vetted by two coinvestigators for retinal hemorrhage by an open-source image processing software, Fiji (National Institutes of Health, USA). ${ }^{36}$ The parameters measured were surface area of retinal hemorrhage in the unit measurement of pixels at $100 \%$ image zoom. The ratio of total surface area of retinal hemorrhage to surface area of optic disc was then obtained to minimize variances from the retinal photographs pertaining to different zooms, patient position, dimension of eyeball, and refractive errors. The overall process quantifying the retinal hemorrhage is shown in Figure 2.

The specifications of the DRS used to capture the retinal photographs were:

1. Field of view: $45^{\circ} \times 40^{\circ}$.

2. Non-mydriatic operation: minimum $3.8 \mathrm{~mm}$ pupil size.

3. Fixation target: 7 internal LEDs.

4. Operating distance: $37 \mathrm{~mm}$.

5. Sensor size: 5 MP $(2592 \times 1944)$.

6. Sensor resolution: 48 pixels/degree.

The specifications of the computer monitor used during the quantification of retinal hemorrhage were:

1. Dimensions: $21.5^{\prime}$ (height $10.4^{\prime \prime}$, width $18.7^{\prime}$ ).

2. Display: $100 \%$ brightness, $100 \%$ contrast.

3. Resolution: $1920 \times 1080$.

4. Orientation: Landscape Display HP P22va.

5. Environment: completely dark room.

\section{Biomarkers AGE, SRAGE, NE-CML, and cystatin C}

The serum samples of participants for biomarkers $A G E$, sRAGE, NE-CML and cystatin $\mathrm{C}$ were stored in a $-80^{\circ} \mathrm{C}$ laboratory freezer. Biomarker processing was conducted by a qualified biochemist on a batch-to-batch basis at the end of the study to avoid inter-assay variation. Serum AGE, sRAGE, NE-CML, and cystatin $C$ concentrations were measured in duplicates and quantified by calorimetric method using Enzyme-linked Immunosorbent Assay (ELISA) (TECANInfinite 200 PRO, Männedorf, Zürich, Switzerland). The ELISA kit for 
a

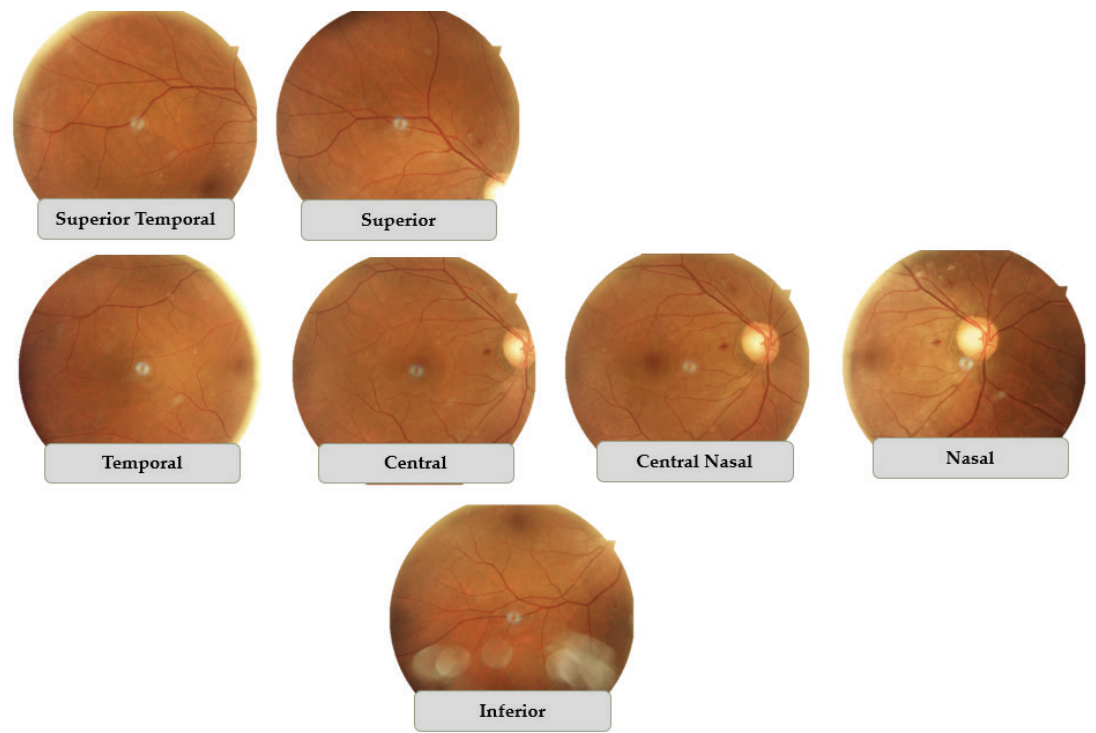

b

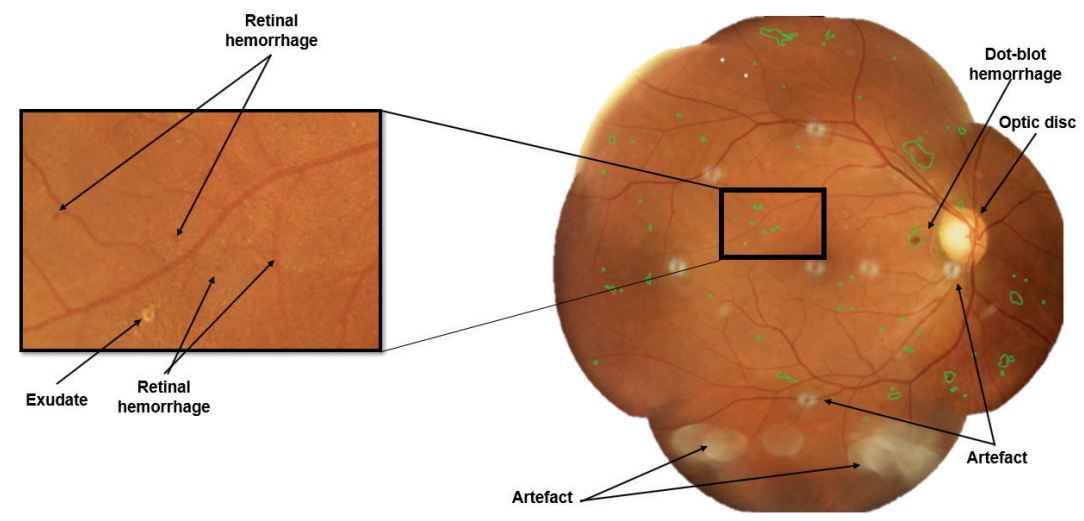

C

$$
\begin{gathered}
\text { Ratio }=(\text { Total Area of Retinal Hemorrhage }) \\
/(\text { Area of Optic Disc })
\end{gathered}
$$

Fig. 2. Overall process to quantify retinal hemorrhages: (a) Seven $45^{\circ}$ retinal image fields taken for the right eye. (b) Retinal map constructed from the 7 individual retinal photographs using i2k Retina ${ }^{\circledR}$ software. Areas of retinal hemorrhage manually quantified (circled in green) with Fiji software. Zoomed-in picture showing small areas of retinal hemorrhage which are difficult to see with the naked eye. (c) Ratio of total area of retinal hemorrhage and area of optic disc obtained to minimize errors pertaining to variance of retinal photographs. 
AGE (Cell Biolabs STA-317, San Diego, CA, USA), N"-CML (Cell Biolabs STA-816, San Diego, CA, USA), sRAGE (Elabscience E-EL-H0295, Houston, TX, USA), and cystatin C (Elabscience E-EL-H0055, Houston, TX, USA) had intra-assay coefficient variances of $4 \%$ and inter-assay coefficient variances of $8 \%$.

\section{HbA1c, BUSE creatinine, liver enzymes, and lipid profile}

A capillary blood sample of $1.5 \mu \mathrm{L}$ was used to measure $\mathrm{HbA} 1 \mathrm{c}$. $\mathrm{HbA} 1 \mathrm{c}$ kits (Alere Afinion, Waltham, MA, USA) had a measuring range of $4-15 \%$ with coefficient variances $(\mathrm{CV})<3 \%$. Serum was procured from participants at baseline and after 8 weeks of supplementation and sent to a national certified pathology lab for general biochemical examination of blood including serum creatinine, blood urea nitrogen (BUN), aspartate aminotransferase (AST), alanine transaminase (ALT), total cholesterol (TC), and high-density lipoproteins (HDL) (ARCHITECT, Abbott Diagnostics, Abbot Park, IL, USA) on the same working day. Coefficient variances for the tests were approximately $<6 \%$.

\section{Statistical analysis}

All statistical analyses were performed using Statistical Package for Social Sciences (SPSS) version 25 (IBM SPSS Inc, Chicago, IL, USA). All data were analyzed by intention-to-treat basis. Each eye was analyzed separately. Probabilities less than $5 \%$ were deemed statistically significant. Baseline characteristics between Tocovid and placebo group were measured using chi-square test for gender; Fisher's exact test for race, smoking status, and grade of DR; Mann-Whitney test for compliance; independent $t$-test for age, duration of T2DM, retinal hemorrhage, glycemic and metabolic parameters, as well as safety tests. ANCOVA, adjusting for baseline values, was used to analyze treatment changes at the end of the study between the intervention and control groups. Odds ratio was calculated for improvement and deterioration in grade of DR for the Tocovid and placebo groups. Correlation between grade of DR with serum biomarkers, retinal hemorrhage, and $\mathrm{HbA} 1 \mathrm{c}$ was analyzed by Spearman's rank correlation.

\section{Results}

\section{Patient characteristics}

Of 118 participants, a total of 43 participants (83 eyes identified with DR) fulfilled the study inclusion and exclusion criteria and were included into the clinical trial. Of these, 21 participants (41 eyes identified with DR) were randomized to the Tocovid group and 22 participants (42 eyes identified with DR) to the placebo group. All participants completed the study duration with no dropouts recorded. All participants and eyes were included for analysis. The flow diagram of participants is shown in Figure 3. 


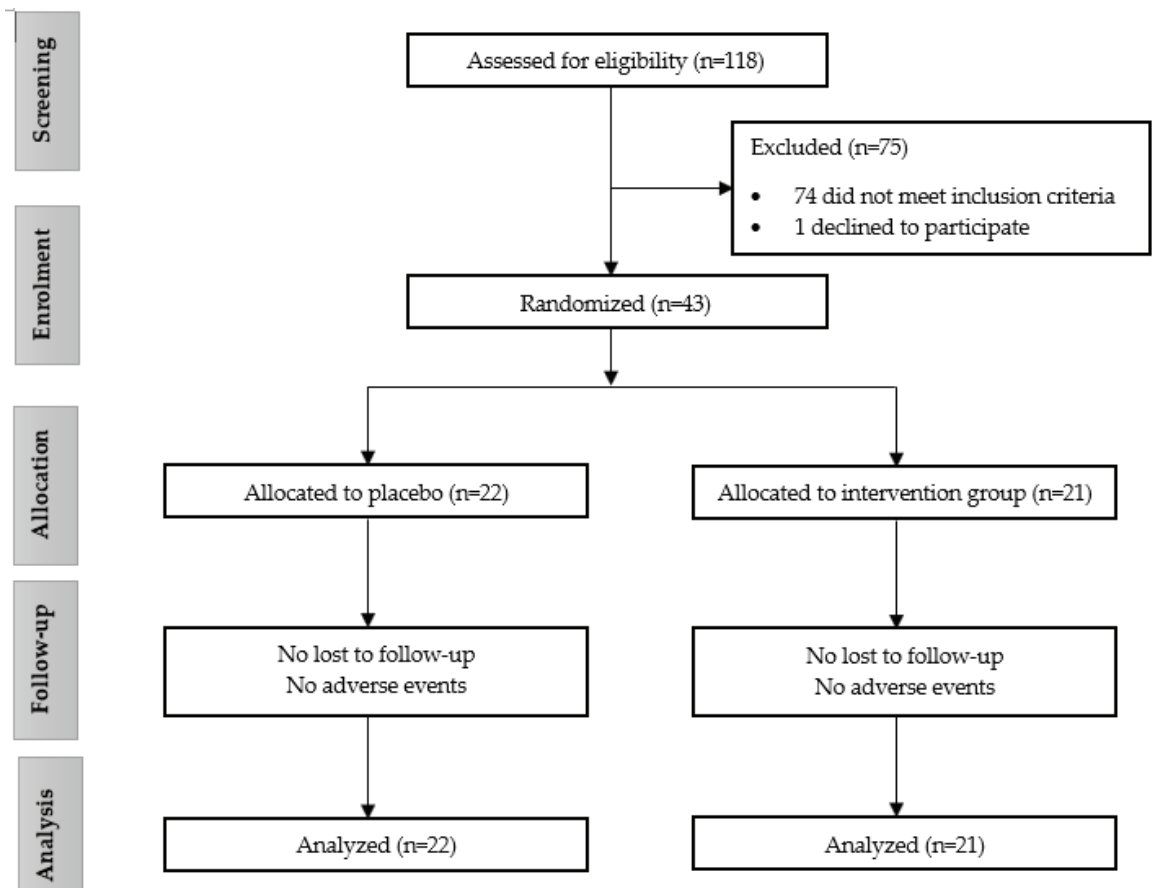

Fig. 3. Flow diagram of participants.

The baseline characteristics of the participants are illustrated in Table 1. The mean participant age was $61.51 \pm 1.25$ years; $74.4 \%$ were male. In terms of ethnicity, $53.5 \%$ of the subjects were Malay, $14 \%$ were Chinese, and $32.6 \%$ were Indian. Both the intervention and control group had statistically similar distributions in age, gender, and ethnicity $(p>0.05)$. The mean duration of T2DM was $18.28 \pm 8.28$ years and there was no significant difference $(p=0.694)$ between intervention and control group. Both groups also had statistically similar prevalence ( $p=0.057$ ) of smoking status. The median (interquartile range, IQR) compliance was $0.98(0.06)$ and $0.95(0.18)$ in the placebo and Tocovid group, respectively, with no statistical differences between both groups $(p=0.123)$.

\section{Baseline ophthalmic parameters and analytes}

The baseline ophthalmic parameters and analytes of the participants are illustrated in Tables 2 and 3, respectively. There were no significant differences in baseline ophthalmic parameters including retinal hemorrhage and grade of DR between intervention and control groups. No statistically significant 
Tocovid and its effects in diabetes and diabetic retinopathy

Table 1. Baseline characteristics of the study population

\begin{tabular}{|c|c|c|c|c|}
\hline & $\begin{array}{l}\text { All } \\
\text { participants } \\
(n=43)\end{array}$ & $\begin{array}{l}\text { Control } \\
(n=22)\end{array}$ & $\begin{array}{l}\text { Intervention } \\
(n=21)\end{array}$ & $p$-Value \\
\hline $\begin{array}{l}\text { Gender }(n, \%): \\
\text { Male } \\
\text { Female }\end{array}$ & $\begin{array}{l}32(74.4) \\
11(25.6)\end{array}$ & $\begin{array}{l}15(68.2) \\
7(31.8)\end{array}$ & $\begin{array}{l}17(81) \\
4(19)\end{array}$ & 0.337 \\
\hline $\begin{array}{l}\text { Race }(\mathrm{n}, \%): \\
\text { Malay } \\
\text { Chinese } \\
\text { Indian }\end{array}$ & $\begin{array}{l}23(53.5) \\
6(14) \\
14(32.6)\end{array}$ & $\begin{array}{l}12(54.5) \\
5(22.7) \\
5(22.7)\end{array}$ & $\begin{array}{l}11(52.4) \\
1(4.8) \\
9(42.9)\end{array}$ & 0.165 \\
\hline $\begin{array}{l}\text { Smoking status (n, \%) } \\
\text { Non-smoker } \\
\text { Current smoker } \\
\text { Ex-smoker }\end{array}$ & $\begin{array}{l}23(54.8) \\
6(14.3) \\
13(31)\end{array}$ & $\begin{array}{l}11(50) \\
1(4.5) \\
10(45.5)\end{array}$ & $\begin{array}{l}12(60) \\
5(25) \\
3(15)\end{array}$ & 0.057 \\
\hline Age (years)* & $61.51 \pm 1.25$ & $63.14 \pm 8.59$ & $59.81 \pm 7.60$ & 0.187 \\
\hline Duration of T2DM (years)* & $18.28 \pm 8.28$ & $18.77 \pm 7.20$ & $17.76 \pm 9.44$ & 0.694 \\
\hline Compliance $^{* *}$ & $0.96(0.11)$ & $0.98(0.06)$ & $0.95(0.18)$ & 0.123 \\
\hline
\end{tabular}

T2DM: type 2 diabetes mellitus

*Data presented as mean \pm standard deviations.

**Data presented as median (interquartile range).

Data not significant at $p>0.05$. 
Table 2. Baseline ophthalmic parameters of Tocovid and placebo groups

\begin{tabular}{|c|c|c|c|c|c|c|}
\hline & \multicolumn{3}{|c|}{$\begin{array}{l}\text { Right eye } \\
(n=41)\end{array}$} & \multicolumn{3}{|l|}{$\begin{array}{l}\text { Left eye } \\
(n=42)\end{array}$} \\
\hline & $\begin{array}{l}\text { Placebo } \\
(n=21)\end{array}$ & $\begin{array}{l}\text { Tocovid } \\
(n=20)\end{array}$ & $p$-Value & $\begin{array}{l}\text { Placebo } \\
(n=22)\end{array}$ & $\begin{array}{l}\text { Tocovid } \\
(n=20)\end{array}$ & $p$-Value \\
\hline $\begin{array}{l}\text { Grade of DR }(n, \%) \\
\text { NDR } \\
\text { Mild NPDR } \\
\text { Moderate NPDR } \\
\text { Severe NPDR } \\
\text { PDR }\end{array}$ & $\begin{array}{l}3(14.3) \\
10(47.6) \\
6(28.6) \\
1(4.8) \\
1(4.8)\end{array}$ & $\begin{array}{l}4(9.8) \\
5(12.2) \\
8(19.5) \\
1(2.4) \\
2(4.9)\end{array}$ & 0.662 & $\begin{array}{l}3(13.6) \\
10(45.5) \\
6(27.3) \\
2(9.1) \\
1(4.5)\end{array}$ & $\begin{array}{l}1(5.0) \\
8(40.0) \\
7(35.0) \\
1(5.0) \\
3(15.0)\end{array}$ & 0.637 \\
\hline $\begin{array}{l}\text { Retinal hemor- } \\
\text { rhage }(D A)^{*}\end{array}$ & $\begin{array}{l}0.74 \pm \\
0.84\end{array}$ & $\begin{array}{l}1.01 \pm \\
1.10\end{array}$ & 0.398 & $\begin{array}{l}0.78 \pm \\
0.94\end{array}$ & $\begin{array}{l}0.99 \pm \\
1.16\end{array}$ & 0.532 \\
\hline
\end{tabular}

DA: disc area; NDR: no diabetic retinopathy; NPDR: non-proliferative diabetic retinopathy; PDR: proliferative diabetic retinopathy

*Data presented as mean \pm standard deviations.

Data not significant at $p>0.05$. 
Tocovid and its effects in diabetes and diabetic retinopathy

Table 3. Baseline analytes of Tocovid and placebo groups

\begin{tabular}{|c|c|c|c|}
\hline & $\begin{array}{l}\text { Placebo } \\
(n=22)\end{array}$ & $\begin{array}{l}\text { Tocovid } \\
(n=21)\end{array}$ & $p$-Value \\
\hline HbA1c (\%) & $8.69 \pm 1.74$ & $9.38 \pm 2.05$ & 0.240 \\
\hline $\begin{array}{l}\text { Blood pressure } \\
\text { SBP }(\mathrm{mmHg}) \\
\text { DBP }(\mathrm{mmHg})\end{array}$ & $\begin{array}{l}136.84 \pm 13.85 \\
77.09 \pm 8.71\end{array}$ & $\begin{array}{l}134.75 \pm 18.06 \\
75.38 \pm 8.67\end{array}$ & $\begin{array}{l}0.671 \\
0.522\end{array}$ \\
\hline BMI (kg/m2) & $27.58 \pm 4.23$ & $28.11 \pm 4.19$ & 0.677 \\
\hline $\begin{array}{l}\text { Safety tests } \\
\text { Urea }(\mathrm{mmol} / \mathrm{L}) \\
\text { Creatinine }(\mu \mathrm{mol} / \mathrm{L}) \\
\text { eGFR }(\mathrm{ml} / \mathrm{min} / 1.73 \mathrm{~m} 2) \\
\text { Total } \mathrm{chol}(\mathrm{mmol} / \mathrm{L}) \\
\text { HDL }(\mathrm{mmol} / \mathrm{L}) \\
\text { AST }(\mathrm{IU} / \mathrm{L}) \\
\text { ALT }(\mathrm{IU} / \mathrm{L})\end{array}$ & $\begin{array}{l}6.10 \pm 4.53 \\
108.05 \pm 52.52 \\
68.23 \pm 26.68 \\
4.49 \pm 1.11 \\
1.18 \pm 0.21 \\
20.27 \pm 12.31 \\
21.50 \pm 14.68\end{array}$ & $\begin{array}{l}6.14 \pm 3.62 \\
111.28 \pm 51.23 \\
67.81 \pm 22.98 \\
4.62 \pm 0.94 \\
1.18 \pm 0.25 \\
25.48 \pm 10.70 \\
27.95 \pm 14.86\end{array}$ & $\begin{array}{l}0.372 \\
0.839 \\
0.956 \\
0.675 \\
0.972 \\
0.148 \\
0.160\end{array}$ \\
\hline $\begin{array}{l}\text { Serum biomarkers } \\
\text { AGE }(\mu \mathrm{g} / \mathrm{mL}) \\
\text { sRAGE }(\mathrm{pg} / \mathrm{mL}) \\
\mathrm{N} \varepsilon-C M L(\mu \mathrm{g} / \mathrm{mL}) \\
\text { Cystatin } C(\mathrm{ng} / \mathrm{mL})\end{array}$ & $\begin{array}{l}109.50 \pm 140.02 \\
978.82 \pm 516.85 \\
1.91 \pm 2.38 \\
1835.25 \pm 958.60\end{array}$ & $\begin{array}{l}117.06 \pm 167.85 \\
1261.53 \pm 869.54 \\
2.04 \pm 2.41 \\
2113.26 \pm 897.79\end{array}$ & $\begin{array}{l}0.874 \\
0.199 \\
0.859 \\
0.333\end{array}$ \\
\hline
\end{tabular}

AGE: advanced glycation end product; ALT: alanine aminotransferase; AST: aspartate aminotransferase; BMI: body mass index; DA: disc area; DBP: diastolic blood pressure; eGFR: estimated glomerular filtration rate; HbA1c: hemoglobin A1c; HDL: high density lipoprotein; NDR: no diabetic retinopathy; Nع-CML: N-epsilon carboxymethyl lysine; NPDR: non-proliferative diabetic retinopathy; PDR: proliferative diabetic retinopathy; SBP: systolic blood pressure; sRAGE: soluble receptor for AGE; Total chol: total cholesterol All values are presented as means \pm standard deviations.

Data not significant at $p>0.05$. 
differences were observed for baseline analytes such as $\mathrm{HbA} 1 \mathrm{c}$, blood pressure, $\mathrm{BMI}$, biomarkers $\mathrm{AGE}$, sRAGE, $\mathrm{N} \varepsilon-\mathrm{CML}$, and cystatin $\mathrm{C}$. In addition, there were no significant differences in baseline safety tests, including renal profile, lipid profile, and liver enzymes between both groups.

\section{Changes in metabolic, ophthalmic, biomarkers, and safety test parame- ters after $\mathbf{8}$ weeks of placebo and Tocovid}

Table 4 compares the treatment changes between placebo and Tocovid group at the end of 8 weeks while controlling for the respective baseline values. Tocovid significantly reduced retinal hemorrhage in the right eye $(-0.24 \mathrm{DA} \pm 0.11, p=$ 0.038 ). While there was reduction in intraretinal hemorrhage in the left eye in both groups, the mean difference was not significant $(p=0.498)$ between both groups. However, the improvement in retinal hemorrhage did not correspond to significant changes $(p=0.05)$ in level of ETDRS grading of DR between both groups (Table 5).

For liver enzymes, Tocovid significantly improved AST compared to placebo $(-8.01 \mathrm{IU} / \mathrm{L} \pm 2.56, p=0.003)$. In addition, ALT was significantly reduced in Tocovid compared to placebo $(-6.01 \mathrm{IU} / \mathrm{L} \pm 2.00, p=0.005)$. There was, however, no significant difference in biomarkers $A G E$, sRAGE, $N \varepsilon-C M L$, and cystatin $C$ between the Tocovid and placebo groups. In addition, no significant differences $(p>0.05)$ were found in $\mathrm{HbA} 1 \mathrm{c}$, mean blood pressure, $\mathrm{BMI}$, and safety tests including renal and lipid profile between both groups after 8 weeks of supplementation with either Tocovid or placebo.

\section{Correlation between grade of DR with retinal hemorrhage and serum biomarkers}

Correlation analysis between grade of DR with retinal hemorrhage and serum biomarkers are presented in Tables 6 and 7. At baseline, there was a highly significant, strong positive correlation between grade of DR and retinal hemorrhage in both eyes (right eye: $r=0.676, p<0.001$; left eye: $r=0.772, p<0.001$ ). The same finding was found at the end of the study. At the 8-week trial period, there was still a highly significant, strong positive correlation between grade of DR and retinal hemorrhage in both eyes (right eye: $r=0.773, p<0.001$; left eye: $r=$ $0.708, p<0.001)$. These highly significant results indicated that the area of retinal hemorrhage was significantly higher in subjects with increasing severity of DR, as assessed by ETDRS grading.

In addition, at baseline and at the end of the study there was a significant, weak positive correlation between grade of $\mathrm{DR}$ in the right eye and serum $\mathrm{N} \varepsilon-\mathrm{CML}$ (baseline: $r=0.342, p=0.028$; end of study: $r=0.379, p=0.015$ ). However, this correlation was not seen in the left eye. In addition, no significant differences between biomarkers AGE, RAGE, and cystatin C and grades of DR were found. 
Table 4. Adjusted changes in metabolic, ophthalmic, biomarkers, and safety test parameters after 8 weeks of placebo and Tocovid

\begin{tabular}{|c|c|c|c|c|}
\hline Parameters & $\begin{array}{l}\text { Placebo } \\
(n=22)\end{array}$ & $\begin{array}{l}\text { Tocovid } \\
(n=21)\end{array}$ & $\begin{array}{l}\text { Mean } \\
\text { difference } \\
(95 \% \mathrm{CI})\end{array}$ & $p$-Value \\
\hline $\begin{array}{l}\text { Retinal hemorrhage (DA) } \\
\text { Right eye } \\
\text { Left eye }\end{array}$ & $\begin{array}{l}0.61 \pm 0.08 \\
0.50 \pm 0.08\end{array}$ & $\begin{array}{l}0.37 \pm 0.08 \\
0.58 \pm 0.09\end{array}$ & $\begin{array}{l}-0.24 \pm 0.11 \\
0.09 \pm 0.12\end{array}$ & $\begin{array}{l}0.038^{*} \\
0.498\end{array}$ \\
\hline HbA1c (\%) & $8.80 \pm 0.17$ & $8.67 \pm 0.18$ & $-0.13 \pm 0.25$ & 0.605 \\
\hline $\begin{array}{l}\text { Blood pressure }(\mathrm{mmHg}) \\
\text { SBP } \\
\text { DBP }\end{array}$ & $\begin{array}{l}131.65 \pm 2.08 \\
74.78 \pm 1.98\end{array}$ & $\begin{array}{l}133.48 \pm 2.12 \\
76.37 \pm 2.02\end{array}$ & $\begin{array}{l}1.83 \pm 2.97 \\
1.59 \pm 2.83\end{array}$ & $\begin{array}{l}0.542 \\
0.578\end{array}$ \\
\hline BMI $\left(\mathrm{kg} / \mathrm{m}^{2}\right)$ & $27.67 \pm 0.24$ & $28.17 \pm 0.24$ & $0.50 \pm 0.34$ & 0.150 \\
\hline $\begin{array}{l}\text { Serum biomarkers } \\
\text { AGE }(\mu \mathrm{g} / \mathrm{mL}) \\
\text { sRAGE }(\mathrm{pg} / \mathrm{mL}) \\
\mathrm{N} \varepsilon-\mathrm{CML}(\mu \mathrm{g} / \mathrm{mL}) \\
\text { Cystatin } \mathrm{C}(\mathrm{ng} / \mathrm{mL})\end{array}$ & $\begin{array}{l}92.47 \pm 27.76 \\
1293.27 \pm \\
152.87 \\
1.53 \pm 0.40 \\
2019.80 \pm \\
172.16 \\
\end{array}$ & $\begin{array}{l}79.09 \pm 29.18 \\
1247.49 \pm \\
156.54 \\
1.64 \pm 0.41 \\
2156.09 \pm \\
176.26 \\
\end{array}$ & $\begin{array}{l}-13.53 \pm 40.28 \\
-45.78 \pm \\
221.01 \\
0.11 \pm 0.57 \\
136.29 \pm \\
247.81 \\
\end{array}$ & $\begin{array}{l}0.739 \\
0.837 \\
0.850 \\
0.585\end{array}$ \\
\hline $\begin{array}{l}\text { Safety tests } \\
\text { Urea }(\mathrm{mmol} / \mathrm{L}) \\
\text { Creatinine }(\mu \mathrm{mol} / \mathrm{L}) \\
\text { eGFR }(\mathrm{ml} / \mathrm{min} / 1.73 \mathrm{~m} 2) \\
\text { Total chol }(\mathrm{mmol} / \mathrm{L}) \\
\text { HDL }(\mathrm{mmol} / \mathrm{L}) \\
\text { AST }(\mathrm{IU} / \mathrm{L}) \\
\text { ALT }(\mathrm{IU} / \mathrm{L})\end{array}$ & $\begin{array}{l}6.44 \pm 0.29 \\
108.45 \pm 2.43 \\
68.86 \pm 1.66 \\
4.47 \pm 0.13 \\
1.12 \pm 0.03 \\
25.96 \pm 1.77 \\
27.47 \pm 1.38\end{array}$ & $\begin{array}{l}6.45 \pm 0.30 \\
107.69 \pm 2.50 \\
69.34 \pm 1.70 \\
4.59 \pm 0.13 \\
1.13 \pm 0.03 \\
17.95 \pm 1.81 \\
21.46 \pm 1.41\end{array}$ & $\begin{array}{l}0.00 \pm 0.42 \\
-0.77 \pm 3.48 \\
0.49 \pm 2.38 \\
0.12 \pm 0.18 \\
-0.043 \pm 0.04 \\
-8.01 \pm 2.56 \\
-6.01 \pm 2.00\end{array}$ & $\begin{array}{l}0.997 \\
0.827 \\
0.839 \\
0.515 \\
0.239 \\
0.003^{*} \\
0.005^{*}\end{array}$ \\
\hline
\end{tabular}

AGE: advanced glycation end product; ALT: alanine aminotransferase; AST: aspartate aminotransferase; BMI: body mass index; DA: disc area; DBP: diastolic blood pressure; eGFR: estimated glomerular filtration rate; $\mathrm{HbA1c}$ : hemoglobin A1c; HDL: high density lipoprotein; NDR: no diabetic retinopathy; Nع-CML: N-epsilon carboxymethyl lysine; NPDR: non-proliferative diabetic retinopathy; PDR: proliferative diabetic retinopathy; SBP: systolic blood pressure; sRAGE: soluble receptor for AGE; Total chol: total cholesterol

All values are presented as means \pm standard errors of mean.

Data adjusted for baseline values.

*Significant at $\mathrm{p}<0.05$. 


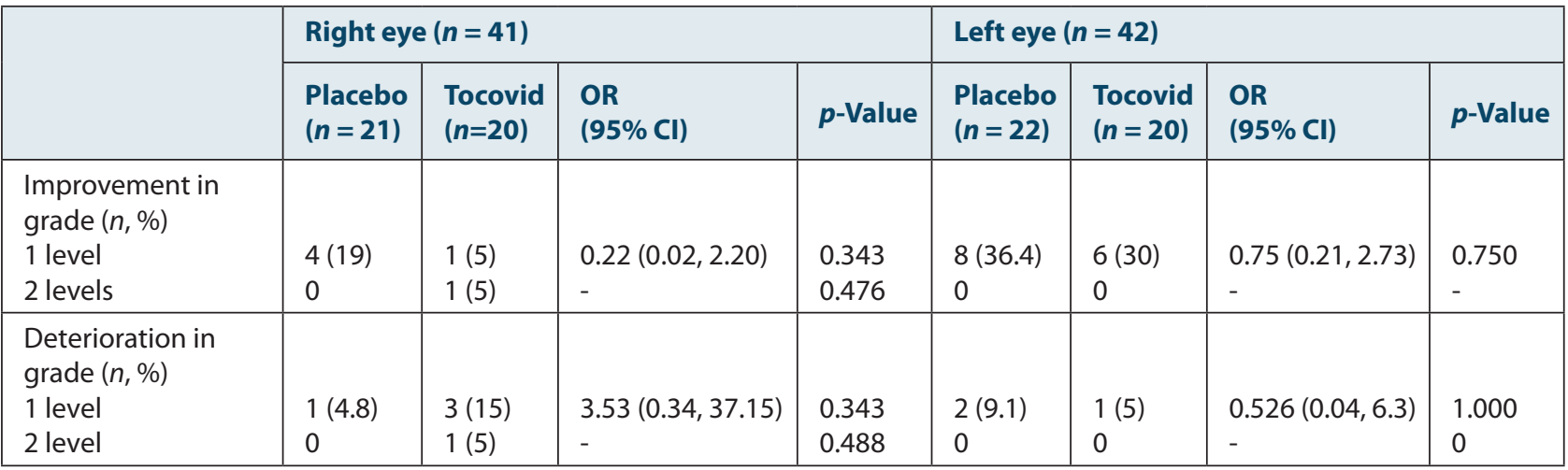

Data not significant at $\boldsymbol{p}>\mathbf{0 . 0 5}$. 


\begin{tabular}{|c|c|c|c|c|c|c|}
\hline \multirow[t]{2}{*}{ Baseline parameters } & \multicolumn{2}{|l|}{$\begin{array}{l}\text { Right eye, } \\
\text { Grade of DR }\end{array}$} & \multicolumn{2}{|l|}{$\begin{array}{l}\text { Left eye, } \\
\text { Grade of DR }\end{array}$} & \multicolumn{2}{|l|}{ HbA1c } \\
\hline & Correlation, $r$ & $p$-Value & Correlation, $r$ & $p$-Value & Correlation, $r$ & $p$-Value \\
\hline $\begin{array}{l}\text { Retinal hemorrhage } \\
\text { (DA) }\end{array}$ & 0.676 & $0.000^{* *}$ & 0.772 & $0.000^{* *}$ & & \\
\hline $\mathrm{HbA1c}$ & 0.309 & $0.049^{*}$ & 0.283 & 0.073 & - & - \\
\hline $\begin{array}{l}\text { Serum biomarkers } \\
\text { AGE }(\mu \mathrm{g} / \mathrm{mL}) \\
\text { sRAGE }(\mathrm{pg} / \mathrm{mL}) \\
\mathrm{N} \varepsilon-\mathrm{CML}(\mu \mathrm{g} / \mathrm{mL}) \\
\text { Cystatin } \mathrm{C}(\mathrm{ng} / \mathrm{mL})\end{array}$ & $\begin{array}{l}0.148 \\
0.123 \\
0.342 \\
0.064\end{array}$ & $\begin{array}{l}0.361 \\
0.444 \\
0.028^{*} \\
0.693\end{array}$ & $\begin{array}{l}0.153 \\
0.173 \\
0.232 \\
0.132\end{array}$ & $\begin{array}{l}0.339 \\
0.274 \\
0.139 \\
0.406\end{array}$ & $\begin{array}{l}0.246 \\
0.361 \\
0.055 \\
0.078\end{array}$ & $\begin{array}{l}0.117 \\
0.017^{*} \\
0.780 \\
0.617\end{array}$ \\
\hline
\end{tabular}

AGE: advanced glycation end product; DA: disc area; HbA1c: hemoglobin A1c; Ne-CML: N-epsilon carboxymethyl lysine; sRAGE: soluble receptor for AGE

*Significant at $p<0.05$.

**Significant at $p<0.001$. 


\begin{tabular}{|l|l|l|l|l|l|l|}
\hline \multirow{2}{*}{$\begin{array}{l}\text { End of study } \\
\text { parameters }\end{array}$} & \multicolumn{2}{l|}{$\begin{array}{l}\text { Right eye, } \\
\text { Grade of DR }\end{array}$} & \multicolumn{2}{l|}{$\begin{array}{l}\text { Left eye, } \\
\text { Grade of DR }\end{array}$} & \multicolumn{2}{l|}{ HbA1c } \\
\cline { 2 - 7 } & Correlation, $\boldsymbol{r}$ & $\boldsymbol{p}$-Value & Correlation, $\boldsymbol{r}$ & $\boldsymbol{p}$-Value & Correlation, $\boldsymbol{r}$ & $\boldsymbol{p}$-Value \\
\hline Retinal hemorrhage (DA) & 0.773 & $0.000^{* *}$ & 0.708 & $0.000^{* *}$ & - & - \\
\hline HbA1c & 0.283 & 0.073 & 0.313 & 0.044 & - & - \\
\hline Serum biomarkers & & & & & & \\
AGE $(\mu \mathrm{g} / \mathrm{mL})$ & 0.179 & 0.276 & 0.204 & 0.207 & -0.201 & 0.207 \\
SRAGE $(\mathrm{pg} / \mathrm{mL})$ & -0.017 & 0.915 & -0.092 & 0.563 & 0.214 & 0.167 \\
Ne-CML $(\mu \mathrm{g} / \mathrm{mL})$ & 0.379 & $0.015^{*}$ & 0.252 & 0.108 & 0.113 & 0.472 \\
Cystatin C $(\mathrm{ng} / \mathrm{mL})$ & -0.140 & 0.383 & -0.004 & 0.978 & 0.142 & 0.362 \\
\hline
\end{tabular}

AGE: advanced glycation end product; DA: disc area; HbA1c: hemoglobin A1c; Nع-CML: N-epsilon carboxymethyl lysine; sRAGE: soluble receptor for $A G E$

*Significant at $p<0.05$.

**Significant at $p<0.001$. 
Lastly, there was a borderline significant, weak positive correlation between grade of DR in the right eye and HbA1c at baseline $(r=0.309, p=0.049)$. However, the correlation between grade of DR and $\mathrm{HbA1c}$ was neither seen at the end of the study or in the left eye. At baseline, biomarker sRAGE also showed a significant weak positive correlation with $\mathrm{HbA1c}(r=0.361 ; p=0.017)$. However, this same correlation was not seen at the end of the 8-week trial period. Otherwise, our findings showed no significant correlation between biomarkers and $\mathrm{HbA} 1 \mathrm{c}$.

\section{Discussion}

This clinical study shows that 8-week supplementation with Tocovid compared to placebo significantly improved the area of retinal hemorrhage in the right eye in patients with DR (Table 2). To the best of our knowledge, this is a new finding, as there are articles describing the benefits of tocotrienol in improving oxidative stress and other metabolic parameters, but literature on its role in DR is limited. In fact, only two animal studies suggest tocotrienol as a potential retinoprotective agent and an inhibitor of angiogenesis. ${ }^{15,16}$ This finding suggests the potential role of tocotrienol as a safe and non-invasive adjunct to the current management of DR. This is particularly important because, other than good glycemic control, treatment options for DR, which include photocoagulation, intraocular injections with antivascular endothelial growth factor, and surgery, are quite limited, costly, and can be debilitating. . $^{37,38}$

The novel technique to quantify area of retinal hemorrhage from digital retinal photographs was developed and performed to assess the disease severity of DR in addition to the established conventional ETDRS grading. This is because the natural history and progression of DR spans many years and by reverse inference, any changes in the grade of DR by Tocovid may only become apparent after a considerable amount of time..$^{39,40}$ Hence, with the help of modern technology and high-resolution displays, this new technique was developed to accurately identify and quantify areas of retinal hemorrhage on the digital retinal photographs at zoomed-in views. This includes very early changes in the retina including small areas of hemorrhage which would normally remain unnoticed to the naked eye of the assessor (Fig. 2b). In order to establish the validity of this technique, correlation analysis between the area of retinal hemorrhage and gold-standard ETDRS grading of DR was performed. There was a highly significant $(p<0.001)$ strong positive correlation between area of retinal hemorrhage and grade of DR in both right and left eyes at baseline and at 8 weeks post-intervention (Tables 6 and 7). This strong correlation endorses the validity of this new technique as an alternative method of assessing disease severity of DR.

However, if retinal hemorrhage strongly correlated with the grade of DR and if Tocovid was able to significantly reduce retinal hemorrhage, why was Tocovid not 
able to cause a corresponding change to the grade of DR? This is because, other than retinal hemorrhage, there are other components such as exudates, venous beading, and neovascularization involved in the ETDRS grading of DR. ${ }^{35}$ As previously mentioned, the pathogenesis of DR spans years. Thus, a longer treatment period with Tocovid would likely be needed for an effect to these other components to occur, thereby allowing a change to the ETDRS grade of DR. On the same account, a longer treatment duration is likely required for a significant improvement in retinal hemorrhage to occur in both eyes due to the protracted nature of DR. Therefore, while the findings of this study report a potential breakthrough in the treatment of DR, more extensive and lengthy research should be conducted to affirm this finding.

In addition, the results of this study demonstrate that 8 weeks of Tocovid supplementation compared to placebo caused a significant improvement in liver enzymes AST and ALT (Table 4). A literature review on this subject matter reveals an increasing interest in tocotrienol and its role as a hepatoprotective agent. Animal studies have shown that tocotrienols are preferentially distributed to the liver. ${ }^{41}$ Here, it acts as an antioxidant by reducing lipid peroxidation as well as an anti-inflammatory agent by the inhibition of the NF-kB pathway, thereby attenuating steatosis in the liver. ${ }^{41}$ Further clinical studies demonstrate the role of tocotrienol in improving liver conditions, including reduced liver echogenicity ${ }^{42}$ and liver stiffness ${ }^{43}$ in patients with non-alcoholic fatty liver disease (NAFLD). NAFLD is associated with metabolic syndrome, which includes DM, hypertension, and obesity, which are very relevant in our patient cohort. ${ }^{44}$ Other than controlling for risk factors, there is no specific pharmacologic treatment available for NAFLD.4 Hence, it would be worthwhile for further investigation to be conducted to verify the benefits of tocotrienol on the liver and if it would be a suitable pharmacological treatment for NAFLD.

However, the results of this trial report no significant improvement in serum biomarkers $\mathrm{AGE}$, SRAGE, $\mathrm{N} \varepsilon-\mathrm{CML}$, and cystatin $\mathrm{C}$ as well other parameters including $\mathrm{HbA} 1 \mathrm{c}$, blood pressure, BMI, total cholesterol, and HDL-cholesterol despite 8-weeks of high-dose Tocovid (Table 4). This is in contrast to previous studies that reported an improvement in AGE and oxidative stress as well as glycemic and metabolic parameters as early as 2 months. ${ }^{9-12,17}$ The lack of effect of Tocovid may be attributed to our study criterion, which required T2DM patients with DR, a complication which conventionally takes years of poor glycemic control to develop. The mean duration of T2DM of our participant pool at 18.28 years \pm 8.28 years was significantly longer than previous studies which recruited participants or animal models with newly diagnosed DM. Studies report that tissues glycated during chronic hyperglycemia can persist in diabetic organs and cause harm for a protracted duration of time..$^{45,46}$ 
The DCCT and UKPDS trials, which showed the importance of tight glycemic control in reducing diabetic-related complications, had recruited patients with newly diagnosed DM. When both trials ended, the patients were followed up for another 10 years. The post-trials showed that, despite achieving similar glycemic control for the next 10 years, patients who were formerly in the control group still had significantly higher rates of diabetic-related complications. ${ }^{20-25}$ Interestingly, the Action in Diabetes and Vascular Disease (ADVANCE), Veteran Administrations Diabetes Trial (VADT), and Action to Control Cardiovascular Risk in Diabetes (ACCORD) trials also reported that intensive glycemic control had minimal effect in preventing diabetes complications. These three trials had recruited patients with long-standing DM at 8,10 , and 11.5 years respectively. ${ }^{47-49}$ Hence, it can be inferred that chronic hyperglycemia had caused sustained harm or a bad "metabolic memory". In other words, even after glucose normalization, there was persistent diabetic vascular stress which led to complications and caused the patients to be refractory to treatment. ${ }^{50,51}$ Thus, the previous studies were able to show improvement in AGE and metabolic parameters of DM because metabolic memory had yet to develop and the effects of chronic hyperglycemia could still be reversed. In our study, chronic exposure to hyperglycemia had led to formation of metabolic memory, causing the lack of treatment effect of tocotrienol.

However, tocotrienol had nonetheless, significantly improved retinal hemorrhage and liver enzymes. This suggests an alternate pathway used by tocotrienol to improve these parameters. More extensive research is warranted to investigate this pathway.

On another note, the results of our trial show that serum levels of biomarker $\mathrm{N} \varepsilon-C M L$ significantly correlates with the presence and progressive severity of DR of the right eye at baseline and at the end of the 8-week treatment period. In addition, the levels of $\mathrm{N} \varepsilon-\mathrm{CML}$ did not correlate with $\mathrm{HbA} 1 \mathrm{c}$, indicating that it is an independent predictor of DR. This positive correlation between $N \varepsilon-C M L$ and DR is congruent with other similar cross-sectional studies. ${ }^{20,26-28}$ This finding is potentially important as current diagnosis of DR is conducted by either of two techniques: traditional ophthalmoscopic examination, which has a wide sensitivity of $27-82 \%$ depending on the training background of the examiner, or retinal photography and grading, which has better sensitivity of $87-100 \%$ and specificity of $83-96 \%$ but requires equipment and manpower, thus limiting the populace that can be effectively screened..$^{52}$ Hence, it would be of great significance if a simple blood test for a biomarker such as $\mathrm{N} \varepsilon-C M L$ can be identified to aid in the diagnosis of DR, rather like how brain natriuretic peptide (BNP) is used as an indicator of heart failure in replacement of physical examination and echocardiography. ${ }^{53}$ 
One of the limitations of this study is its small sample size. Only 43 subjects out of the intended 52 required for an adequate sample size were recruited due to financial limitations. This may have reduced the statistical power of the study and therefore, the lack of effect of Tocovid compared to placebo on the outcomes of the study. Moreover, the treatment duration of 8 weeks was likely too short for most changes, especially long-lasting biomarkers and ophthalmic parameters, to take into effect. Future studies with a longer treatment duration are warranted. Another limitation is the long duration of T2DM among the recruited participant cohort. Although DR takes years of poor glycemic control to develop, future studies directed at newly diagnosed DR patients should be considered. Finally, measurement of baseline and end of study vitamin $E$ is warranted for future studies to negate the potential confounding effects of vitamin E on the study results.

\section{Conclusion}

In conclusion, 8 weeks of Tocovid supplementation was able to improve the area of retinal hemorrhage and liver enzymes ALT and AST, suggesting its role as an adjunct to the current treatment of DR and possibly NAFLD. The novel method of quantifying retinal hemorrhage strongly correlated with grade of DR and can potentially be used to identify early changes in the retina in response to treatment. Serum Ne-CML was found to correlate with grade of DR independent of $\mathrm{HbA} 1 \mathrm{c}$ levels. This indicates its potential role as a biomarker for DR.

\section{Declarations}

\section{Author contributions}

KAK and YC designed the pilot study. YC, SMQT, and BA performed the clinical trial and collected the data. SEK graded the retinal photographs. YC analyzed the data and prepared the manuscript. KAK and BA reviewed and edited the manuscript.

\section{Competing interests}

The authors declare no conflict of interest. The funders had no role in the design of the study, the collection, analyses, or interpretation of data, writing of the manuscript, or in the decision to publish the results.

\section{Funding}

This work was supported by Monash University Malaysia under the Tropical Medicine and Biology (TMB) grant (Grant number: TMB-2018-CR3185140918-SuzTMQ/KAK) and ExcelVite, Malaysia. In addition, the use of the i $2 \mathrm{k}^{\text {Retina }}{ }^{\circledR}$ software was sponsored by Dual Align (NY, USA). 


\section{Acknowledgements}

We would like to extend our gratitude to ExcelVite (Malaysia) for subsidizing the cost of Tocovid Suprabio. In addition, we would like to thank Dual Align (NY, USA) for sponsoring the use of their i $2 k$ Retina $^{\circledR}$ software. We would like to thank the following staff at the Monash University Clinical Research Centre for their technical assistance: Noraskin Mohamad, Ungku Zulaikha, Chui Chor Sin, Pang Pei Ling, and Savithri Gopal. Finally, we would like to thank Cheng Hong Sheng for analyzing the serum biomarkers.

\section{References}

1. Yau JW, Rogers SL, Kawasaki R, et al. Global prevalence and major risk factors of diabetic retinopathy. Diabetes Care. 2012;35(3):556-564.

2. Bourne RR, Stevens GA, White RA, et al. Causes of vision loss worldwide, 1990-2010: a systematic analysis. Lancet Glob Health. 2013;1(6):e339-349.

3. Congdon NG, Friedman DS, Lietman T. Important causes of visual impairment in the world today. JAMA. 2003;290(15):2057-2060.

4. Montonen J, Knekt P, Järvinen R, Reunanen A. Dietary antioxidant intake and risk of type 2 diabetes. Diabetes Care. 2004 Feb;27(2):362-366.

5. Suksomboon N, Poolsup N, Sinprasert S. Effects of vitamin E supplementation on glycaemic control in type 2 diabetes: systematic review of randomized controlled trials. J Clin Pharm Ther. 2011;36(1):53-63.

6. Xu R, Zhang S, Tao A, Chen G, Zhang M. Influence of vitamin E supplementation on glycaemic control: a meta-analysis of randomised controlled trials. PLoS One. 2014;9(4):e95008.

7. Peh HY, Tan WD, Liao W, Wong WF. Vitamin E therapy beyond cancer: Tocopherol versus tocotrienol. Pharmacol Ther. 2016;162:152-169.

8. Nazaimoon WW, Sakinah O, Gapor A, Khalid B. Effects of palm olein tocopherol and tocotrienol on lipid peroxidation, lipid profiles and glycemic control in non-insulin diabetes mellitus patients. Nutr Res. 1996;16(11-12):1901-1911.

9. Nazaimoon WW, Khalid B. Tocotrienols-rich diet decreases advanced glycosylation endproducts in non-diabetic rats and improves glycemic control in streptozotocin-induced diabetic rats. Malays J Pathol. 2002;24(2):77-82.

10. Budin SB, Othman F, Louis SR, Bakar MA, Das S, Mohamed J. The effects of palm oil tocotrienolrich fraction supplementation on biochemical parameters, oxidative stress and the vascular wall of streptozotocin-induced diabetic rats. Clinics. 2009;64(3):235-244.

11. Kanaya Y, Doi T, Sasaki H, et al. Rice bran extract prevents the elevation of plasma peroxylipid in KKAy diabetic mice. Diabetes Res Clin Prac. 2004;66: S157-S160.

12. Matough FA, Budin SB, Hamid ZA, Abdul-Rahman M, Al-Wahaibi N, Mohammed J. Tocotrienolrich fraction from palm oil prevents oxidative damage in diabetic rats. Sultan Qaboos Univ Med J. 2014;14(1): e95-103.

13. Pushparajah E, Rajadurai M. Effects of processing on the content and composition of tocopherols and tocotrienols in palm oil. International Conference on Palm Oil Product Technology in the Eighties; 1981 Jun 22-24; Special - Malaysian Agri (501), Kuala Lumpur: The Incorporated Society of Planters Malaysia; 1983.

14. Hashimoto T, Kato A, Tanabe K, et al. Studies on tocopherols and tocotrienols in Malaysian palm oil. Proceedings of International Symposium of the Advanced Industrial Utilisation of the Tropical Plants; 1980 Sep 1-4; Tsubuka, Japan: International Research and Development Cooperation Division, Ministry of International Trade and Industry; 1980. 
15. Chida M, Suzuki K, Nakanishi-Ueda T, et al. In vitro testing of antioxidants and biochemical end-points in bovine retinal tissue. Ophthalmic Res. 1999;31(6):407-415.

16. Nakagawa K, Shibata A, Yamashita S, et al. In vivo angiogenesis is suppressed by unsaturated vitamin E, tocotrienol. J Nutr. 2007;137(8):1938-43.

17. Cheng HS, Ton SH, Tan JB, Abdul Kadir KJ. The ameliorative effects of a tocotrienol-rich fraction on the AGE-RAGE axis and hypertension in high-fat-diet-fed rats with metabolic syndrome. Nutrients. 2017;9(9):984.

18. Nowotny K, Jung T, Höhn A, Weber D, Grune T. Advanced glycation end products and oxidative stress in type 2 diabetes mellitus. Biomolecules. 2015;5(1):194-222.

19. Tarr JM, Kaul K, Chopra M, Kohner EM, Chibber R. Pathophysiology of diabetic retinopathy. ISRN Ophthalmol. 2013;2013:343560.

20. Genuth S, Sun W, Cleary P, et al. Glycation and carboxymethyllysine levels in skin collagen predict the risk of future 10-year progression of diabetic retinopathy and nephropathy in the diabetes control and complications trial and epidemiology of diabetes interventions and complications participants with type 1 diabetes. Diabetes. 2005;54(11):3103-3111.

21. Monnier VM, Bautista O, Kenny D, et al. Skin collagen glycation, glycoxidation, and crosslinking are lower in subjects with long-term intensive versus conventional therapy of type 1 diabetes: relevance of glycated collagen products versus $\mathrm{HbA} 1 \mathrm{c}$ as markers of diabetic complications. DCCT Skin Collagen Ancillary Study Group. Diabetes Control and Complications Trial. Diabetes. 1999;48(4):870-80.

22. The Diabetes Control and Complications Trial Research Group. The effect of intensive treatment of diabetes on the development and progression of long-term complications in insulindependent diabetes mellitus. N Engl J Med. 1993;1993(329):977-986.

23. The Diabetes Control and Complications Trial/Epidemiology of Diabetes Interventions and Complications (DCCT/EDIC) Study Research Group. Intensive diabetes treatment and cardiovascular disease in patients with type 1 diabetes. N Engl J Med. 2005;353(25):2643-2653.

24. Holman RR, Paul SK, Bethel MA, Matthews DR, Neil HA. 10-year follow-up of intensive glucose control in type 2 diabetes. N Engl J Med. 2008;359(15):1577-1589.

25. King P, Peacock I, Donnelly R. The UK Prospective Diabetes Study (UKPDS): clinical and therapeutic implications for type 2 diabetes. Br J Clin Pharmacol. 2001;48(5):643-648.

26. Choudhuri S, Dutta D, Sen A, et al. Role of N-epsilon-carboxy methyl lysine, advanced glycation end products and reactive oxygen species for the development of nonproliferative and proliferative retinopathy in type 2 diabetes mellitus. Mol Vis. 2013;19:100-113.

27. Boehm B, Schilling S, Rosinger S, et al. Elevated serum levels of Ne-carboxymethyl-lysine, an advanced glycation end product, are associated with proliferative diabetic retinopathy and macular oedema. Diabetologia. 2004;47(8):1376-1379.

28. Mishra N, Saxena S, Shukla RK, et al. Association of serum N $\varepsilon$-Carboxy methyl lysine with severity of diabetic retinopathy. J Diabetes Complications. 2016;30(3):511-517.

29. Kerkeni M, Saïdi A, Bouzidi H, Yahya SB, Hammami M. Elevated serum levels of AGEs, sRAGE, and pentosidine in Tunisian patients with severity of diabetic retinopathy. Microvasc Res. 2012;84(3):378-383.

30. Ng ZX, Chua KH, Iqbal T, Kuppusamy UR. Soluble receptor for advanced glycation end-product (sRAGE)/pentosidine ratio: a potential risk factor determinant for type 2 diabetic retinopathy. Int J Mol Sci. 2013;14(4):7480-7491.

31. Miranda ER, Fuller JK, Perkins R, et al. Soluble RAGE Sequesters Advanced Glycation End Products following an Overnight Fast in T1DM Patients. Diabetes. 2018;16(1).

32. Campion CG, Sanchez-Ferras O, Batchu SN. Potential role of serum and urinary biomarkers in diagnosis and prognosis of diabetic nephropathy. Can J Kidney Health Dis. 2017;4:1-18. 
33. Khatami PG, Soleimani A, Sharifi N, Aghadavod E, Asemi Z. The effects of high-dose vitamin E supplementation on biomarkers of kidney injury, inflammation, and oxidative stress in patients with diabetic nephropathy: a randomized, double-blind, placebo-controlled trial. J Clin Lipidol. 2016;10(4):922-929.

34. Chen J, Ausayakhun S, Ausayakhun S, et al. Comparison of autophotomontage software programs in eyes with CMV retinitis. Invest Ophthalmol Vis Sci. 2011;52(13):9339-9344.

35. Wilkinson CP, Ferris FL, Klein RE, et al. Proposed international clinical diabetic retinopathy and diabetic macular edema disease severity scales. Ophthalmology. 2003;110(9):1677-1682.

36. Schindelin J, Arganda-Carreras I, Frise E, et al. Fiji: an open-source platform for biological-image analysis. Nat Methods. 2012;9(7):676-682.

37. Nathan DM, Buse JB, Davidson MB, et al. Medical management of hyperglycemia in type 2 diabetes: a consensus algorithm for the initiation and adjustment of therapy: a consensus statement of the American Diabetes Association and the European Association for the Study of Diabetes. Diabetes Care. 2009;32(1):193-203.

38. Jones S, Edwards RT. Diabetic retinopathy screening: a systematic review of the economic evidence. Diabet Med. 2010;27(3):249-256.

39. Klein R, Klein BE, Moss SE, Cruickshanks KJ. The Wisconsin Epidemiologic Study of diabetic retinopathy: XIV. Ten-year incidence and progression of diabetic retinopathy. Arch Ophthalmol. 1994;112(9):1217-1228.

40. Klein R, Klein BE, Moss SE, Cruickshanks KJ. The Wisconsin epidemiologic study of diabetic retinopathy: XVII: The 14-year incidence and progression of diabetic retinopathy and associated risk factors in type 1 diabetes. Ophthalmology. 1998;105(10):1801-1805.

41. Muto C, Yachi R, Aoki Y, Koike T, Igarashi O, Kiyose C. Gamma-tocotrienol reduces the triacylglycerol level in rat primary hepatocytes through regulation of fatty acid metabolism. J Clin Biochem Nutr. 2013;52(1):32-37.

42. Magosso E, Ansari MA, Gopalan Y, et al. Tocotrienols for normalisation of hepatic echogenic response in nonalcoholic fatty liver: a randomised placebo-controlled clinical trial. Nutr J. 2013;12(1):166.

43. Leong WH. Tocotrienol: the crown jewel in Malaysian red palm oil. Alternative Medicine. 2018;24:16-9

44. Vernon G, Baranova A, Younossi Z. Systematic review: the epidemiology and natural history of non囚alcoholic fatty liver disease and non囚alcoholic steatohepatitis in adults. Aliment Pharmacol Ther. 2011;34(3):274-285.

45. Rosca MG, Mustata TG, Kinter MT, Ozdemir AM, Kern TS. Glycation of mitochondrial proteins from diabetic rat kidney is associated with excess superoxide formation. Am J Physiol Renal Physiol. 2005;289(2):F420-F430.

46. Nowotny K, Jung T, Höhn A, Weber D, Grune T. Advanced glycation end products and oxidative stress in type 2 diabetes mellitus. Biomolecules. 2015;5(1):194-222.

47. The ADVANCE Collaborative Group. Intensive blood glucose control and vascular outcomes in patients with type 2 diabetes. N Engl J Med. 2008;358:2560-2572.

48. The Action to Control Cardiovascular Risk in Diabetes Study Group. Effects of intensive glucose lowering in type 2 diabetes. N Engl J Med. 2008;358:2545-2559.

49. Duckworth W, Abraira C, Moritz T, et al. Glucose control and vascular complications in veterans with type 2 diabetes. N Engl J Med. 2009;360(2):129-139.

50. Ceriello A. The emerging challenge in diabetes: the "metabolic memory". Vasc Pharmacol. 2012;57(5-6):133-138.

51. Aschner PJ, Ruiz AJ. Metabolic memory for vascular disease in diabetes. Diabetes Technol Ther. 2012;14(S1):S68-S74.

52. Torok Z, Peto T, Csosz E, et al. Tear fluid proteomics multimarkers for diabetic retinopathy screening. BMC Ophthalmol. 2013;13(1):40. 
Chiew et al.

53. Troughton RW, Frampton CM, Yandle TG, Espine EA, Nicholls MG, Richards AM. Treatment of heart failure guided by plasma aminoterminal brain natriuretic peptide (N-BNP) concentrations. Lancet. 2000;355(9210):1126-1130. 